\title{
On a Limiting Procedure for Obtaining Physical States for an Infinite Fermi System
}

\author{
G. FANO ${ }^{\star}$ \\ Istituto di Fisica dell'Università di Bologna, Bologna, Italia
}

Received June 16, 1971

\begin{abstract}
We propose a limiting procedure for obtaining physical states for an infinite non-relativistic Fermi system. We take the thermodynamic limit of vector states in the Fock representation of the C.A.R. algebra, representing a condensate state of "atoms" each of which is formed by 4 fermions. In a simplified example considered in detail, the limit state has a simple decomposition into the product of two B.C.S. states. If $B^{+}$is the operator creating the "atom" from the vacuum $\left|\psi_{O_{F}}\right\rangle$, it is proved that the states obtained by taking the thermodynamic limit of the vector states corresponding to $\left(B^{+}\right)^{n}\left|\psi_{0 F}\right\rangle$ and $\sum_{n=0}^{\infty} 3^{n / 2}\left(B^{+}\right)^{n} /(n !)^{2}\left|\psi_{0, F}\right\rangle$ respectively, coincide on the gauge-invariant elements of the algebra for a suitable value of $z$.
\end{abstract}

\section{Introduction}

We shall present here a procedure for obtaining states over the algebra of the anticommutation relations (C.A.R. algebra) which are locally normal, translation invariant and not quasi-free. We recall that a "quasifree" state or "generalized free" state is characterized by the property that its truncated $(n, m)$-point functions $W_{n, m}^{T}$ vanish if $n+m>2$.

In the simple case considered in this paper, the state of the system belongs to the closed-convex hull of the set of the quasi-free states when the thermodynamic limit is performed. However, the procedure we propose is of a completely general character, and it is probable that new physical states may be found working along the lines of the present work.

Our state is constructed by means of the following procedure ${ }^{1}$ :

i) We include the one-dimensional system in a finite box of linear dimension $L$.

* Partially supported by C.N.R.

1 This procedure has been already indicated in the unpublished report [1]. The idea of including four-particle correlations in a Fermi system is quite old: see, e.g. Flowers [2] and Bremond and Valatin [3]. However, to the best of our knowledge, no workable example of physical state for the infinite system which includes four-particle correlations is known. The state proposed in ref. [3] is a product state of finite dimensional type in Powers terminology [4]. Hence by Theorem 5.20 of Ref. [4], this state becomes quasifree in the thermodynamic limit if we require translation invariance. 
ii) We consider a suitable sequence $\varrho_{L, N}$ of vector states in the Fock representation of the C.A.R. algebra; these states $\varrho_{L, N}$ represent a physical situation with $N / 4$ condensed "atoms", each of which is formed by 4 fermions.

iii) We take the thermodynamic limit, in the weak topology, of the sequence $\varrho_{L, N}$ (in other words, we let $L \rightarrow \infty, N \rightarrow \infty$, while the density $N / L$ is equal to a constant value $d$, and we compute the limit of the $(n, m)$-point correlation functions $\left.W_{n, m}\right)$.

The methods we shall use are adapted from statistical mechanics and have been successfully tested [5] in the elementary case of the B.C.S. state. In this case the "atoms" mentioned are replaced by Cooper pairs. It follows (see Ref. [5]) that the thermodynamic limit of the sequence $\left\{\varrho_{L, N}\right\}$ is the gauge-invariant B.C.S. state ${ }^{2}$. It is well known that this state can be decomposed into the integral $\int_{0}^{2 \pi} \frac{d \theta}{2 \pi}$ of gaugedependent B.C.S. states over the phase $\theta$ of the gauge group [7] [8]. Furthermore the gauge-dependent B.C.S. state is the thermodynamic limit of a vector state arising from the vector $e^{B^{+}}\left|\psi_{0, F}\right\rangle$, where $B^{+}$is the operator creating a "Cooper pair" and $\left|\psi_{0, F}\right\rangle$ is the Fock vacuum.

In our case, too, the thermodynamic limit of the states $\varrho_{L, N}$ can be decomposed into the integral $\int_{0}^{2 \pi} \frac{d \theta}{2 \pi}$ of gauge-dependent states. However, since now the "atoms" are not pairs of particles but are composed of four particles, it turns out that these gauge-dependent states arise from the vector $\sum_{n=0}^{\infty} \frac{\left(B^{+}\right)^{n}}{(n !)^{2}}\left|\psi_{0, F}\right\rangle$.

The form chosen for the operator $B^{+}$has been suggested by the necessity of simplifying the calculations. With this choice (see Eqs. (6), (12)), it turns out that in the thermodynamic limit the state $\varrho_{L, N}$ becomes simply the product of two gauge-invariant B.C.S. states. Probably more interesting states may be obtained by taking more general form of the operator $B^{+}$.

\section{The Canonical State}

We use the same notations as in Ref. [5]. The field operators in the Fock representation $\pi_{F}^{L}$ of the C.A.R. algebra $\mathfrak{A}(L)$ constructed over the Hilbert space $\mathscr{L}^{2}(L)$ are $a_{F}(f)$ and its hermitian adjoint $a_{F}(f)^{+}$. Hence heuristically they are defined by ${ }^{3}$ :

$$
a_{F}(f)=\int_{L} a_{F}(x) f(x) d x ; \quad a_{F}(f)^{+}=\int_{L} a_{F}(x)^{+} \overline{f(x)} d x
$$

${ }^{2}$ Of course this result has long been known to physicists. See for instance Ref. [6].

${ }^{3}$ We indicate with the same symbol the "box" $L$ and its linear measure. 
where $a_{F}(x)$ is the Fermion "field", $f \in \mathscr{L}^{2}(L),\left|\psi_{0, F}^{L}\right\rangle$ denotes the Fock vacuum and $H_{F}^{L}$ denotes the Hilbert space of the representation $\pi_{F}^{L}$. Finally we set:

$$
f_{j}(x)=\frac{1}{\sqrt{L}} e^{i k_{j} x}, x \in L, k_{j}=j \frac{2 \pi}{L} ; \quad j=0, \pm 1, \pm 2 \ldots
$$

and

$$
a_{k_{j}}=a_{F}\left(f_{j}\right)
$$

The operator that creates from the Fock vacuum $\left|\psi_{0, F}^{L}\right\rangle$ the "atom" of particles mentioned in the introduction, is defined by ${ }^{4}$ :

$$
B_{4, L}^{+}=\frac{1}{4} \sum_{j=-\infty}^{+\infty} \sum_{j^{\prime}=-\infty}^{+\infty} c\left(k_{j}, k_{j^{\prime}}\right) a_{k_{j}}^{+} a_{-k_{j}}^{+} a_{k^{\prime}}^{+} a_{-k_{j^{\prime}}}^{+}
$$

(the label 4 indicates that $B_{4, L}^{+}$creates 4 particles).

Without loss of generality, we can assume that $c$ fulfils the conditions:

$$
c(k, h)=c(h, k) ; \quad c(-k, h)=c(k,-h)=-c(k, h) .
$$

Hence $B_{4, L}^{+}$can be written simply as:

$$
B_{4, L}^{+}=\sum_{j=1}^{+\infty} \sum_{j^{\prime}=1}^{+\infty} c\left(k_{j}, k_{j^{\prime}}\right) a_{k_{j}}^{+} a_{-k_{j}}^{+} a_{k^{\prime}}^{+} a_{-k_{j^{\prime}}}^{+}
$$

(A sufficient condition for the operator $B_{4, L}^{+}$to be bounded is

$$
\left.\sum_{j=1}^{\infty} \sum_{j^{\prime}=1}^{\infty}\left|c\left(k_{j}, k_{j^{\prime}}\right)\right|<\infty\right) .
$$

We define now a sequence $\left\{v_{4, L}^{(n)}\right\}$ of vectors $v_{4, L}^{(n)} \in H_{F}^{L}$ by:

$$
\left|v_{4, L}^{(n)}\right\rangle=\frac{1}{\sqrt{(2 n) ! n !}}\left(B_{4, L}^{+}\right)^{n}\left|\psi_{0, F}^{L}\right\rangle \text { for } n=0,1,2 \ldots
$$

Let $N_{o p}$ be the total number operator. Then each vector $\left|v_{4, L}^{(n)}\right\rangle$ is an eigenstate of $N_{o p}$ corresponding to the eigenvalue $N=4 n$. Furthermore $\left|v_{4, L}^{(n)}\right\rangle$ defines a vector state $\varrho_{L, N}$ on the algebras $\mathfrak{A}\left(L^{\prime}\right)\left(L^{\prime} \cong L\right)$ by the formula:

$$
\varrho_{L, N}(A)=\frac{\left\langle v_{4, L}^{(n)}\left|\pi_{F}^{L}(A)\right| v_{4, L}^{(n)}\right\rangle}{\left\langle v_{4, L}^{(n)} \mid v_{4, L}^{(n)}\right\rangle} ; \quad A \in \mathfrak{A}\left(L^{\prime}\right) .
$$

${ }^{4}$ While the author was using more complicated operators $B_{4, L}^{+}$creating four particles in a state with zero total momentum, G. Gallavotti suggested the simple form (4) for the operator $B_{4, L}^{+}$. 
The limit state for the infinite system is given by (when the thermodynamic limit exists and is unique):

$$
\left.\begin{array}{rl}
\varrho_{B_{4}, d}= & \text { weak limit } \varrho_{L, N} \\
& L \rightarrow \infty \\
& N \rightarrow \infty
\end{array}\right\} N / L=d
$$

and is defined on all the local algebras $\mathfrak{U}\left(L^{\prime}\right)$.

For reasons that will be apparent later, we shall call $\varrho_{B_{4}, d}$ the canonical state.

We already know an elementary case in which the limit (9) exists: assume that the function $c$ is of the form:

$$
c(k, h)=\xi(k) \xi(h) \quad \text { where } \quad \xi(-k)=-\xi(k) .
$$

In this case $B_{4, L}^{+}=\left(B_{2, L}^{+}\right)^{2}$, where:

$$
B_{2, L}^{+}=\sum_{j=1}^{\infty} \xi\left(k_{j}\right) a_{k_{j}}^{+} a_{-k_{j}}^{+} .
$$

As said in the Introduction, the thermodynamic limit of the sequence $\varrho_{L, N}$ is equal to the gauge-invariant B.C.S. state, for large classes of functions $\xi$. Let us now take for $c$ the more general function:

$$
c(r, s)=\xi_{1}(r) \xi_{1}(s)+\xi_{2}(r) \xi_{2}(s)
$$

where the functions $\xi_{1}, \xi_{2}$ have disjoint supports $E_{1}, E_{2}$. Furthermore the functions $\xi_{1}, \xi_{2}$ are supposed to be sufficiently regular for the results of [5] to be applicable.

Hence we have:

$$
B_{4, L}^{+}=\left(B_{1}^{+}\right)^{2}+\left(B_{2}^{+}\right)^{2}
$$

where both $B_{1}^{+}$and $B_{2}^{+}$are of the form (11), i.e.:

$$
B_{i}^{+}=\sum_{j=1}^{\infty} \xi_{i}\left(k_{j}\right) a_{k_{j}}^{+} a_{-k_{j}}^{+} ; \quad i=1,2 .
$$

Since $E_{1}$ and $E_{2}$ are disjoint, we have:

$$
\left[B_{1}, B_{2}^{+}\right]_{-}=\left[B_{2}, B_{1}^{+}\right]_{-}=0
$$

and of course:

$$
\left[B_{1}^{+}, B_{2}^{+}\right]_{-}=0 \text {. }
$$

Let us compute the norm of $\left|v_{4, L}^{(n)}\right\rangle$. Using Eqs. (13), (15), (16) and taking into account the property that the Fock state is a product state [4] with respect to any orthogonal decomposition of the one-particle 
Hilbert space $\mathscr{L}^{2}(L)$, we have:

$$
\begin{aligned}
&\left\langle v_{4, L}^{(n)} \mid v_{4, L}^{(n)}\right\rangle=\frac{1}{(2 n) !(n !)^{2}}\left\langle\psi_{0, F}^{L}\left|B_{4, L}^{n}\left(B_{4, L}^{+}\right)^{n}\right| \psi_{0, F}^{L}\right\rangle \\
&=\frac{1}{(2 n) !(n !)^{2}} \sum_{s=0}^{n} \sum_{s^{\prime}=0}^{n}\left(\begin{array}{l}
n \\
s
\end{array}\right)\left(\begin{array}{l}
n \\
s^{\prime}
\end{array}\right)\left\langle\psi_{0, F}^{L}\left|B_{1}^{2 s}\left(B_{1}^{+}\right)^{2 s^{\prime}} B_{2}^{2(n-s)}\left(B_{2}^{+}\right)^{2\left(n-s^{\prime}\right)}\right| \psi_{0, F}^{L}\right\rangle \\
&=\frac{1}{(2 n) !(n !)^{2}} \sum_{s=0}^{n} \sum_{s^{\prime}=0}^{n}\left(\begin{array}{l}
n \\
s
\end{array}\right)\left(\begin{array}{l}
n \\
s^{\prime}
\end{array}\right)\left\langle\psi_{0, F}^{L}\left|B_{1}^{2 s}\left(B_{1}^{+}\right)^{2 s^{\prime}}\right| \psi_{0, F}^{L}\right\rangle \\
& \cdot\left\langle\psi_{0, F}^{L}\left|B_{2}^{2(n-s)}\left(B_{2}^{+}\right)^{2\left(n-s^{\prime}\right)}\right| \psi_{0, F}^{L}\right\rangle \\
&= \frac{1}{(2 n) !(n !)^{2}} \sum_{s=0}^{n}\left(\begin{array}{l}
n \\
s
\end{array}\right)^{2}\left\langle\psi_{0, F}^{L}\left|B_{1}^{2 s}\left(B_{1}^{+}\right)^{2 s}\right| \psi_{0, F}^{L}\right\rangle \\
& \cdot\left\langle\psi_{0, F}^{L}\left|B_{2}^{2(n-s)}\left(B_{2}^{+}\right)^{2(n-s)}\right| \psi_{0, F}^{L}\right\rangle .
\end{aligned}
$$

It is convenient to define the quantities:

$$
a_{n}^{(i) L}=\sum_{0<j_{1}<j_{2} \cdots<j_{n}} \xi_{i}\left(k_{j_{1}}\right)^{2} \xi_{i}\left(k_{j_{2}}\right)^{2} \ldots \xi_{i}\left(k_{j_{n}}\right)^{2} \text { for } i=1,2 .
$$

Setting $a_{4, n}^{L}=\left\langle v_{4, L}^{(n)} \mid v_{4, L}^{(n)}\right\rangle$ and using Eq. (17) of [5], we have:

where:

$$
a_{4, n}^{L}=\sum_{s=0}^{n} B^{L}(s)
$$

$$
B^{L}(s)=\frac{1}{(2 n) !(n !)^{2}}\left(\begin{array}{l}
n \\
s
\end{array}\right)^{2}(2 s) !^{2}(2 n-2 s) !^{2} a_{2 s}^{(1) L} a_{2 n-2 s}^{(2) L} .
$$

Let us denote by $T$. $\lim _{N}$. the thermodynamic limit with $N \rightarrow \infty, L \rightarrow \infty$, $\frac{N}{L}=d$ $\frac{N}{L}=d$, and let us define:

$$
d_{1}(s)=\frac{4 s}{L} ; \quad d_{2}(s)=\frac{4 n-4 s}{L}=d-d_{1}(s)
$$

$g_{1}\left(d_{1}\right)=T . \lim _{\frac{4 s}{L}=d_{1}} \frac{1}{L} \log a_{2 s}^{(1) L} ; \quad g_{2}\left(d_{2}\right)=T . \lim _{\frac{4 n-4 s}{L}=d_{2}} \frac{1}{L} \log a_{2 n-2 s}^{(2) L}$

$$
\jmath_{1}\left(d_{1}\right)=e^{-2 \frac{d g_{1}}{d d_{1}}} ; \quad 3_{2}\left(d_{2}\right)=e^{-2 \frac{d g_{2}}{d d_{2}}}
$$

We know that the limits $g_{1}$ and $g_{2}$ exist and are differentiable functions of the densities $d_{1}, d_{2}$ respectively, for all $0<d_{1}<d_{1, \max }, 0<d_{2}<d_{2, \max }$ 
(see Ref. [5]). Furthermore it can be easily proved that these limits are uniform in $d_{1}, d_{2}$ [9]. We shall see that an analogous result holds for T. $\lim _{\frac{4 n}{L}=d} \frac{1}{L} \log a_{4, n}^{L}$, for all $0<d<D=$ inf. $\left\{d_{1, \max }, d_{2, \max }\right\}$. We use the maximum term method: let us assume first that $T . \lim _{\frac{4 s}{L}=d_{1}} \frac{1}{L} \log B^{L}(s)$
exists; since:

$$
\max _{0 \leqq s \leqq n} B^{L}(s) \leqq \sum_{s=0}^{n} B^{L}(s) \leqq(n+1) \max _{0 \leqq s \leqq n} B^{L}(s)
$$

we have:

$$
\begin{aligned}
& T \cdot \lim _{\frac{4 n}{L}=d} \frac{1}{L} \log \max _{0 \leqq s \leqq n} B^{L}(s) \leqq T \cdot \lim _{\frac{4 n}{L}=d} \cdot \frac{1}{L} \log a_{4, n}^{L} \\
& \quad \leqq T \cdot \lim _{\frac{4 n}{L}=d} \frac{1}{L} \log \left[(n+1) \max _{0 \leqq s \leqq n} B^{L}(s)\right] \\
& \quad=T \cdot \lim _{\frac{4 n}{L}=d} \frac{1}{L} \log (n+1)+T \cdot \lim _{\frac{4 n}{L}=d} \frac{1}{L} \log \max _{0 \leqq s \leqq n} B^{L}(s) \\
& \quad=T \cdot \lim _{\frac{4 n}{L}=d} \frac{1}{L} \log \max _{0 \leqq s \leqq n} B^{L}(s) .
\end{aligned}
$$

Therefore:

$$
\text { T. } \lim _{\frac{4 n}{L}=d} \frac{1}{L} \log a_{4, n}^{L}=T . \lim _{\frac{4 s^{*}}{L}=d^{*}} \frac{1}{L} \log B^{L}\left(s^{*}\right)
$$

where $s^{*}$ is a value of $s$ where $B^{L}(s)$ is maximum. On the other hand, using Stirling's formula, we can deduce from Eq. (20) that for $0<d<D$

$$
\begin{aligned}
F\left(d_{1}\right) & =T \cdot \lim _{\frac{4 s}{L}=d_{1}} \frac{1}{L} \log B^{L}(s) \\
& =\frac{1}{2}\left[d_{1} \log d_{1}+d_{2} \log d_{2}-d \log \frac{d}{2}\right]+g_{1}\left(d_{1}\right)+g_{2}\left(d_{2}\right) .
\end{aligned}
$$

In order to find the maximum of $B^{L}(s)$ for $0 \leqq s \leqq n$ (or, which is the same, for $0 \leqq d_{1} \leqq d$ ) we set $\frac{d F}{d d_{1}}=0$. Since $d_{2}=d-d_{1}$, we obtain:

$$
2\left(\frac{d g_{1}}{d d_{1}}-\frac{d g_{2}}{d d_{2}}\right)=\log d_{2}-\log d_{1} .
$$


Taking into account Eqs. (23), we have finally:

$$
\frac{d_{1}}{d_{2}}=\frac{3_{1}\left(d_{1}\right)}{3_{2}\left(d_{2}\right)}
$$

Eq. (29), and the condition $d_{1}+d_{2}=d$, constitute a system of two equations in two unknowns. Living aside the discussion of the existence of the solution $\left(d_{1}^{*}, d_{2}^{*}\right)$ of this system, let us assume that such a solution exists and is unique (it is easy to find pairs of functions $\xi_{1}, \xi_{2}$ such that this is the case; we shall see later an example). Then from Eqs. (26), (27), we obtain:

$$
\begin{aligned}
g(d) & =T \cdot \lim _{\frac{4 n}{L}=d} \frac{1}{L} \log a_{4, n}^{L} \\
& =\frac{1}{2}\left[d_{1}^{*} \log d_{1}^{*}+d_{2}^{*} \log d_{2}^{*}-d \log \frac{d}{2}\right]+g_{1}\left(d_{1}^{*}\right)+g_{2}\left(d_{2}^{*}\right) .
\end{aligned}
$$

We shall now prove a simple product property of the $T$. limit of the sequence $\left\{\varrho_{L, 4 n}\right\}$. Let $A_{1}, A_{2}$ denote two elements of the algebra $\mathfrak{A}(L)$, where $A_{i}$ is generated by creation and annihilation operators with momenta belonging to $E_{i}(i=1,2)$.

Taking into account Eqs. (19), (20), and recalling that the Fock state is a product state with respect to any orthogonal decomposition of $\mathscr{L}^{2}(L)$, we have:

$$
\begin{aligned}
& \varrho_{L, 4 n}\left(A_{1} A_{2}\right)=\frac{\left\langle v_{4, L}^{(n)}\left|A_{1} A_{2}\right| v_{4, L}^{(n)}\right\rangle}{\left\langle v_{4, L}^{(n)} \mid v_{4, L}^{(n)}\right\rangle} \\
& =\frac{\sum_{s=0}^{n}\left(\begin{array}{l}
n \\
s
\end{array}\right)^{2}\left\langle\psi_{0, F}^{L}\left|B_{1}^{2 s} A_{1}\left(B_{1}^{+}\right)^{2 s}\right| \psi_{0, F}^{L}\right\rangle\left\langle\psi_{0, F}^{L}\left|B_{2}^{2(n-s)} A_{2}\left(B_{2}^{+}\right)^{2(n-s)}\right| \psi_{0, F}^{L}\right\rangle}{\left\langle v_{4, L}^{(n)} \mid v_{4, L}^{(n)}\right\rangle} \\
& =\frac{\sum_{s=0}^{n} \varrho_{L, 4 s}^{(1)}\left(A_{1}\right) \varrho_{L, 4 n-4 s}^{(2)}\left(A_{2}\right) B^{L}(s)}{\sum_{s=0}^{n} B^{L}(s)}
\end{aligned}
$$

where $\varrho_{L, 4 s}^{(1)}, \varrho_{L, 4 n-4 s}^{(2)}$ denote the vector states arising respectively from $\left(B_{1}^{+}\right)^{2 s}\left|\psi_{0, F}^{L}\right\rangle$ and $\left(B_{2}^{+}\right)^{2(n-s)}\left|\psi_{0, F}^{L}\right\rangle$ (see Eq. (9) of Ref. [5]).

Consider now the function $B^{L}(s)$ : from the existence of the limit (27), and the fact that this limit is a differentiable function of $d_{1}$, it follows (see, e.g. Ref. [10]) that $B^{L}(s)$ has a sharp peak for $s=s^{*}=\frac{L d_{1}^{*}}{4}$, the 
width of the peak being of the $\operatorname{order}^{5} \sqrt{L}$, as is the usual behaviour of the canonical partition function with respect to the number of particles. Since in our case the function $F\left(d_{1}\right)$ corresponds to the free-energy per unit length of a "one-phase state" it follows [10] that for any $\varepsilon>0$

$$
\text { T. } \lim _{\frac{4 n}{L}=d} \frac{\sum_{s \in I_{\varepsilon}} B^{L}(s)}{\sum_{s=0}^{n} B^{L}(s)}=1
$$

where:

$$
I_{\varepsilon}=\left\{s: d_{1}^{*}-\varepsilon<\frac{4 s}{L}<d_{1}^{*}+\varepsilon\right\}
$$

Furthermore we have [5]:

$$
\begin{gathered}
\text { T. } \lim _{\frac{4 s}{L}=d_{1}} \varrho_{L, 4 s}^{(1)}\left(A_{1}\right)=\varrho_{d_{1}}^{(1)}\left(A_{1}\right) \\
T . \lim _{\frac{4 n-4 s}{L}=d_{2}} \varrho_{L, 4 n-4 s}^{(2)}\left(A_{2}\right)=\varrho_{d_{2}}^{(2)}\left(A_{2}\right)
\end{gathered}
$$

where $\varrho_{d_{i}}^{(i)}(i=1,2)$ are two gauge-invariant pure B.C.S. states determined by the relation (see Eq. (36) of [5]):

$$
\varrho_{d_{i}}^{(i)}\left[a(f)^{+} a(g)\right]=\int_{E_{i}} \overline{\tilde{f}(k)} \tilde{g}(k) \frac{\partial_{i}\left(d_{i}\right) \xi_{i}(k)^{2}}{\partial_{i}\left(d_{i}\right) \xi_{i}(k)^{2}+1} d k \text { for } \quad i=1,2 .
$$

From relations (31) through (35) and the fact that the limits (34), (35) are uniform with respect to the densities $d_{i}$, it follows that:

$$
\text { T. } \lim _{\frac{4 n}{L}=d} \varrho_{L, 4 n}\left(A_{1} A_{2}\right)=\varrho_{d_{1}^{*}}^{(1)}\left(A_{1}\right) \varrho_{d_{2}^{*}}^{(2)}\left(A_{2}\right) .
$$

Therefore our state has a simple product decomposition in the $T$. limit: Let us decompose the one-particle Hilbert space $\mathscr{L}^{2}(R)$ into the direct sum of three Hilbert spaces $H_{1}, H_{2}, H_{3}$ that are defined by ${ }^{6}$ :

$$
H_{i}=\left\{f: f \in \mathscr{L}^{2}(R), \quad \text { support } \tilde{f} \cong E_{i}\right\} \quad \text { for } \quad i=1,2,3
$$

where $E_{3}=R-\left(E_{1} \cup E_{2}\right)$ and $\tilde{f}(k)=\frac{1}{\sqrt{2 \pi}} \int_{-\infty}^{+\infty} e^{-i k x} f(x) d x$. Finally let $\mathfrak{A}\left(H_{i}\right)(i=1,2,3)$ denote the C.A.R. algebra over $H_{i}$, and let $\varrho_{0, F}$ denote

${ }^{5}$ Taking $d_{1}$ as independent variable, the width of the peak is of order $1 / \sqrt{L}$, and hence tends to zero in the T.limit.

${ }^{6}$ The sets $E_{i}$ are supposed to be symmetric with respect to the mapping $k \rightarrow-k$. 
the Fock state. From what has been said, it follows that:

$$
\varrho_{B_{4}, d}=\left.\left.\left.\varrho_{d_{1}^{\star}}^{(1)}\right|_{\mathfrak{Y}\left(H_{1}\right)} \otimes \varrho_{d_{2}^{*}}^{(2)}\right|_{\mathfrak{A}\left(H_{2}\right)} \otimes \varrho_{0, F}\right|_{\mathfrak{Q}\left(H_{3}\right)} .
$$

The states $\varrho_{d^{*}}^{(i)}$ can be decomposed into an integral of gauge-dependent B.C.S. states, i.e. (see Eq. (133) of [5]):

$$
\varrho_{d_{z^{*}}}^{(i)}=\int_{0}^{2 \pi} \frac{d \theta_{i}}{2 \pi} \varrho_{\left[z_{i}\left(d^{*}\right), \theta_{2}\right]}^{(i)} \text { for } i=1,2
$$

Hence we have, omitting for simplicity the restrictions to the subalgebras:

$$
\varrho_{B_{4}, d}=\int_{0}^{2 \pi} \frac{d \theta_{1}}{2 \pi} \int_{0}^{2 \pi} \frac{d \theta_{2}}{2 \pi} \varrho_{\left.\left[3_{1}(d)^{*}\right), \theta_{1}\right]}^{(1)} \otimes \varrho_{\left[32\left(d_{2}^{*}\right), \theta_{2}\right]}^{(2)} \otimes \varrho_{0, F} .
$$

Since the product of two quasi-free states is quasi-free, it follows that $\varrho_{B_{4}, d}$ belongs to the closed convex hull of the set of the quasi-free states.

\section{The Grand Canonical State}

As said in the Introduction, also the gauge invariant state $\varrho_{B_{4}, d}$ can be decomposed into the integral $\int_{0}^{2 \pi} \frac{d \theta}{2 \pi}$ of gauge-dependent states. Let us seek these gauge-dependent states.

We know that by taking the thermodynamic limit of the vector states corresponding to $\left(B_{2, L}^{+}\right)^{n}\left|\psi_{0, F}^{L}\right\rangle$ and $\exp \left(\sqrt{3} B_{2, L}^{+}\right)\left|\psi_{0, F}^{L}\right\rangle$ respectively (see Eq. (11)), we obtain the gauge-invariant and the gauge-dependent B.C.S. states for the infinite system. It has been proved in [5] that these states coincide on the gauge-invariant elements of the algebra for an appropriate value of $\mathfrak{z}$, the relation between $\mathfrak{z}$ and $d=\frac{2 n}{L}$ being the same as the relation between activity and density in statistical mechanics.

In order to obtain similar results in the present case, it is necessary to make use of an idea due to G. Gallavotti ${ }^{7}$.

Let us restrict ourselves to the case considered by Eq. (10), and let us define the quantities $a_{2, n}^{L}, b_{4, n}^{L}$ by:

$$
\begin{aligned}
& \left\langle e^{\sqrt{3} B_{2, L}^{+}} \psi_{0, F}^{L} \mid e^{\sqrt{3} B_{2, L}^{+}} \psi_{0, F}^{L}\right\rangle=\sum_{n=0}^{\infty} a_{2, n}^{L} 3^{n} \\
& \left\langle e^{\sqrt{3} B_{4, L}^{+}} \psi_{0, F}^{L} \mid e^{\sqrt{3} B_{4, L}^{+}} \psi_{0, F}^{L}\right\rangle=\sum_{n=0}^{\infty} b_{4, n}^{L} 3^{n} .
\end{aligned}
$$

\footnotetext{
${ }^{7}$ Private communication.
} 
In the analogy with statistical mechanics that has been extensively applied in [5], the expressions (42), (43) play the role of grand partition functions. Hence for the methods of statistical mechanics to be applied, it is necessary that $\log a_{2, n}^{L}, \log b_{4, n}^{L}$ be extensive quantities in the thermodynamic limit. It is indeed true that $\log a_{2, n}^{L}$ is an extensive quantity for large $n, L$. What can we say about $\log b_{4, n}^{L}$ ? We have

$$
\begin{aligned}
b_{4, n}^{L} & =\frac{1}{(n !)^{2}}\left\langle\left(B_{4, L}^{+}\right)^{n} \psi_{0, F}^{L} \mid\left(B_{4, L}^{+}\right)^{n} \psi_{0, F}^{L}\right\rangle \\
& =\frac{1}{(n !)^{2}}\left\langle\left(B_{2, L}^{+}\right)^{2 n} \psi_{0, F}^{L} \mid\left(B_{2, L}^{+}\right)^{2 n} \psi_{0, F}^{L}\right\rangle=\frac{1}{(n !)^{2}}[(2 n) !]^{2} a_{2,2 n}^{L}
\end{aligned}
$$

so that $\frac{1}{n} \log b_{4, n}^{L} \rightarrow \infty$ in the thermodynamic limit, and the analogy with statistical mechanics does not work. In particular it is not true that the states $\left(B_{4, L}^{+}\right)^{n}\left|\psi_{0, F}^{L}\right\rangle$ and $e^{\sqrt{3} B_{4, L}^{+}}\left|\psi_{0, F}^{L}\right\rangle$ become equivalent on the gauge-invariant part of the algebra in the thermodynamic limit.

Then Gallavotti suggested to the author that he consider the vector $|L, \precsim\rangle$ defined by (see Eq. (7)):

$$
|L, \mathfrak{\jmath}\rangle=\sum_{n=0}^{\infty} \frac{\mathfrak{\jmath}^{\frac{n}{2}}}{\sqrt{(2 n) !}} \frac{\left(B_{4, L}^{+}\right)^{n}}{n !}\left|\psi_{0, F}^{L}\right\rangle=\sum_{n=0}^{\infty} \mathfrak{\jmath}^{\frac{n}{2}}\left|v_{4, L}^{(n)}\right\rangle ; \quad \mathfrak{\jmath}>0 .
$$

Hence:

$$
\langle L, \jmath \mid L, \jmath\rangle=\sum_{n=0}^{\infty} \jmath^{n}\left\langle v_{4, L}^{(n)} \mid v_{4, L}^{(n)}\right\rangle=\sum_{n=0}^{\infty} a_{4, n}^{L} \jmath^{n}
$$

and it follows from Eq. (44) that the expression $\log a_{4, n}^{L}=\log b_{4, n}^{L} /(2 n)$ ! is an extensive quantity in the $T$. limit when $c(r, s)=\xi(r) \xi(s)$. Therefore in this case the results of statistical mechanics stating the equivalence of the canonical and grand-canonical ensembles can be applied, so that the vector $|L, z\rangle$ gives rise again to the gauge-invariant B.C.S. state.

Gallavotti conjectured that if $A$ is a gauge-invariant element of the algebra, the state $\varrho_{3}$ defined by:

$$
\varrho_{\mathfrak{\jmath}}(A)=\lim _{L \rightarrow \infty} \frac{\left\langle L, \mathfrak{\jmath}\left|\pi_{F}^{L}(A)\right| L, \mathfrak{\jmath}\right\rangle}{\langle L, \mathfrak{\jmath} \mid L, \mathfrak{\jmath}\rangle}
$$

coincides with $\varrho_{B_{4}, d}$ on the gauge-invariant elements for an appropriate value of $d$, even when the function $c(r, s)$ is not of the form $\xi(r) \xi(s)$. We can easily verify that Gallavotti's conjecture is true in the case considered in the previous section (see Eq. (12)), since the factor ${ }^{8} \frac{1}{\sqrt{(2 n) !} n !}$ in ${ }^{8}$ Of course the factor $\frac{1}{(n !)^{2}}$ works equally well. 
formula (7) assures the existence of the limit $T . \lim _{\frac{4 n}{L}=d} \frac{1}{L} \log a_{4, n}^{L}=g(d)$; generally the function $g$ turns out to be a differentiable concave function of the density and the function $a_{4, n}^{L} z^{n}$ has a sharp peak for $n=d(z) \frac{L}{4}$, where:

$$
d(\jmath)=\lim _{L \rightarrow \infty} \frac{1}{L} \frac{\sum_{n=0}^{\infty} 4 n a_{4, n}^{L} 3^{n}}{\sum_{n=0}^{\infty} a_{4, n}^{L} 3^{n}} .
$$

The inverse function $孔(d)$ of the function $d(z)$ coincides with $e^{-4 \frac{d g}{d d}}$. The limit:

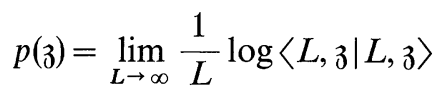

exists for all $\mathfrak{0}$, and we have:

$$
p(\mathfrak{z})=g[d(3)]+\frac{d(3)}{4} \log _{3}
$$

etc. Everything works as in the usual formalism of statistical mechanics [9-11]. For these reasons we call $\varrho_{3}$ the grand-canonical state.

Example. Let $E_{1}, E_{2}$ be the following disjoint subsets of the real line $R$ :

$$
\begin{aligned}
& E_{1}=\left\{k: k \in R, 0 \leqq|k| \leqq \frac{k_{\mathrm{MAX}}}{2}\right\} \\
& E_{2}=\left\{k: k \in R, \frac{k_{\mathrm{MAX}}}{2}<|k| \leqq k_{\mathrm{MAx}}\right\}
\end{aligned}
$$

where $k_{\mathrm{MAX}}$ is a positive number, and let $\xi_{i}$ be the characteristic function of the set $E_{i}(i=1,2)$. Then Eqs. (18), (20) and (22) become:

$$
\begin{gathered}
a_{n}^{(i) L}=\left(\begin{array}{l}
v \\
n
\end{array}\right) \quad \text { where } \quad v=\frac{k_{\mathrm{MAX}}}{2} \cdot \frac{L}{2 \pi} \\
B^{L}(s)=\frac{(v !)^{2}}{(n !)^{2}(2 v-2 n) !}\left(\begin{array}{l}
n \\
s
\end{array}\right)^{2} \frac{\left(\begin{array}{c}
2 v-2 n \\
v-2 s
\end{array}\right)}{\left(\begin{array}{l}
2 n \\
2 s
\end{array}\right)} \\
g_{i}\left(d_{i}\right)=\frac{d_{i}}{2}\left[\alpha_{i} \log \alpha_{i}-\left(\alpha_{i}-1\right) \log \left(\alpha_{i}-1\right)\right]
\end{gathered}
$$


where $\alpha_{i}=\frac{k_{\mathrm{MAX}}}{2 \pi d_{i}}(i=1,2)$. Furthermore ${ }_{\partial_{i}}\left(d_{i}\right)=\left(\alpha_{i}-1\right)^{-1}$. The maximum of $B^{L}(s)$ is reached for $\frac{4 s}{L}=d_{1}^{*}=\frac{d}{2}$, i.e. for $s=\frac{n}{2}$. Putting $s=\frac{n}{2}+t$, the behaviour of $B^{L}(s)$ near the peak is given by:

$$
B^{L}[s(t)] \sim \frac{(v !)^{2}}{(n !)^{2}(2 v-2 n) !} \cdot \frac{2^{2 v-2 n+1}}{\pi \sqrt{(v-n) n}} e^{-\frac{4 t^{2}}{v-n}} .
$$

Since both $v$ and $n$ are proportional to $L$, it follows that the width of the peak is of order $\sqrt{L}$, as it should ${ }^{9}$.

Finally, $g(d)$ is equal to the following differentiable concave function of the density $d$ for all $d<\frac{k_{\mathrm{MAX}}}{\pi}$ :

$$
g(d)=\frac{d}{2} \log \frac{\alpha^{\alpha}}{(\alpha-1)^{\alpha-1}}
$$

where $\alpha=\frac{v}{n}=\frac{k_{\mathrm{MAx}}}{\pi d}$, and:

$$
d(\mathfrak{z})=\frac{k_{\mathrm{MAX}}}{\pi} \frac{\sqrt{3}}{\sqrt{3}+1} .
$$

The correlation functions of the states $\varrho_{B_{4}, d}$ and $\varrho_{3}$ can be easily computed. For instance the Fourier transforms of the $(1,1),(2,0)$ and $(4,0)$-point functions for the state $\varrho_{3}$ are given by:

$$
\begin{aligned}
& \tilde{W}_{1,1}\left(p_{1}, q_{1}\right)=\delta\left(p_{1}-q_{1}\right)\left[\xi_{1}\left(p_{1}\right)+\xi_{2}\left(p_{1}\right)\right] \frac{\pi d}{k_{\mathrm{MAX}}} \\
& \tilde{W}_{2,0}\left(p_{1}, p_{2}\right)=0 \\
& \tilde{W}_{4,0}\left(p_{1}, p_{2}, p_{3}, p_{4}\right) \\
& =\Delta\left[\xi_{1}\left(p_{1}\right) \xi_{1}\left(p_{2}\right) \xi_{1}\left(p_{3}\right) \xi_{1}\left(p_{4}\right)+\xi_{2}\left(p_{1}\right) \xi_{2}\left(p_{2}\right) \xi_{2}\left(p_{3}\right) \xi_{2}\left(p_{4}\right)\right] \frac{\sqrt{3}}{(\sqrt{3}+1)^{2}}
\end{aligned}
$$

where:

$$
\begin{aligned}
\Delta=\delta\left(p_{1}\right. & \left.+p_{2}\right) \delta\left(p_{3}+p_{4}\right) \operatorname{sign}\left(p_{1} p_{3}\right)-\delta\left(p_{1}+p_{3}\right) \delta\left(p_{2}+p_{4}\right) \operatorname{sign}\left(p_{1} p_{2}\right) \\
& +\delta\left(p_{1}+p_{4}\right) \delta\left(p_{2}+p_{3}\right) \operatorname{sign}\left(p_{1} p_{2}\right) .
\end{aligned}
$$

9 The following asymptotic formula can be easily proved:

$$
a_{4, n}^{L}=\frac{(v !)^{2} 2^{2 v-2 n}}{(n !)^{2}(2 v-2 n) ! \sqrt{\pi n}}[1+o(n, v)] .
$$


Since the function $W_{2,0}$ vanishes while $W_{4,0}$ is different from zero, $\varrho_{3}$ is not a quasi-free state. And being the weak limit of a sequence of normal states, $\varrho_{3}$ is locally normal. Translation invariance can be checked from the form of the correlation functions. Finally, defining:

$$
\varrho_{\mathfrak{z}, \theta}(A)=\varrho_{\mathfrak{z}}\left(\tau_{\theta} A\right) \text { for } \quad A \in \mathfrak{A}
$$

where $\tau_{\theta}$ is the automorphism of the algebra generated by the gauge transformation, i.e.:

$$
\tau_{\theta}[a(f)]=e^{i \theta} a(f)
$$

we have:

$$
\varrho_{B_{4}, d}=\int_{0}^{2 \pi} \frac{d \theta}{2 \pi} \varrho_{3, \theta} .
$$

In fact the state on the R.H.S. of Eq. (64) vanishes on the non gaugeinvariant monomials of the algebra and coincides with $\varrho_{z}$ on the gaugeinvariant monomials.

Acknowledgements. The author is greatly indebted to G. Gallavotti, for suggesting the analogy with statistical mechanics, which is fundamental for this work. He is also happy to acknowledge a number of useful discussions with S. Doplicher, J. L. Lebowitz, G. Loupias, R. T. Powers, J. E. Roberts and D. Testard.

\section{References}

1. Fano, G.: Loupias, G., Conjectures on a class of physical states of Fermi systems... (unpublished report).

2. Flowers, B.H.: Proceedings of the Rutherford Jubilee Conference, Manchester (Ed. Birks, J.B.), 207, London 1961.

3. Bremond, B., Valatin, J. G.: Nuclear Physics 41, 640 (1963).

4. Powers, R. T.: Princeton Thesis (1967).

5. Fano, G., Loupias, G.: Commun. math. Phys. 20, 143 (1971).

6. Blatt,J.M.: Theory of superconductivity, New-York-London: Academic Press 1964.

7. Haag, R.: Nuovo Cimento 25, 287 (1962).

8. Fano, G.: Nuovo Cimento 38, 597 (1965).

9. Fisher, M.E.: Arch. Rat. Mech. Anal. 17, 377 (1964).

10. Jones, G. L., Kennedy, R.E.: J. Math. Phys. 8, 460 (1966).

11. Ruelle,D.: Statistical mechanics - rigorous results. New York: W. A. Benjamin, Inc., 1969.

G. Fano

Istituto di Fisica dell'Università di Bologna Bologna, Italy 